\title{
SCIMITAR SYNDROME WITH UNILOBAR RIGHT LUNG- A CASE REPORT
}

\author{
Vathada Nooka Raju1, A. Ayyappa ${ }^{2}$, Banavath Durgaprasad Naik ${ }^{3}$, Veerapaneni Vijaysai ${ }^{4}$, N. S. Vara Prasad ${ }^{5}$
}

1 Professor, Department of Pulmonary Medicine, AMC.

${ }^{2}$ Associate Professor, Department of Pulmonary Medicine, AMC.

3 Postgraduate Student, Department of Pulmonary Medicine, AMC.

${ }^{4}$ Postgraduate Student, Department of Pulmonary Medicine, AMC.

5 Postgraduate Student, Department of Pulmonary Medicine, AMC.

\section{ABSTRACT}

\section{BACKGROUND}

Scimitar syndrome is characterised by hypoplasia and anomalous pulmonary venous drainage of right lung. Because of its rarity, we present a case of a 21-year-old male who came with respiratory tract symptoms and pulmonary arterial hypertension and was diagnosed as Scimitar syndrome with unilobar right lung based on imaging methods and FOB findings.

\section{KEYWORDS}

Scimitar Syndrome, Pulmonary Arterial Hypertension, Hypoplasia.

HOW TO CITE THIS ARTICLE: Raju VN, Ayyappa A, Naik BD, et al. Scimitar syndrome with unilobar right lung- A case report. J. Evolution Med. Dent. Sci. 2017;6(23):1950-1952, DOI: 10.14260/Jemds/2017/427

\section{BACKGROUND}

Scimitar Syndrome (SS) is also known as pulmonary venolobar syndrome or hypogenetic lung syndrome which is a rare congenital condition characterised by partial anomalous pulmonary venous connection of right lung to inferior vena cava, dextroposed heart, right lung hypoplasia. ${ }^{1}$ This anomalous connection occurs most commonly below the diaphragm. It can occur either isolated or with other cardiac malformations like ASD. ${ }^{2}$ Scimitar syndrome will have a varied presentation from asymptomatic to severe symptomatic (pulmonary hypertension, heart failure). ${ }^{3}$ Infants and neonates present with severe symptoms due to the underlying congenital cardiac defects like ASD, whereas adults present with recurrent respiratory tract infections or haemoptysis. ${ }^{4}$ The diagnosis of scimitar syndrome can be made by chest $\mathrm{x}$-ray, 2D echo, and other imaging techniques like contrast CT of lung, CT angiography and bronchoscopy. We present a case of scimitar syndrome with unilobar right lung, who presented at OPD with respiratory tract symptoms and pulmonary arterial hypertension.

\section{Case Report}

A 21-year-old male patient was admitted in our hospital with cough, expectoration, dyspnoea and chest pain since 5 months. SOB was of grade 1, and retrosternal chest pain was dull aching in character without any radiation. There was no other relevant past history, and he was not a smoker or alcoholic. His vitals were within normal limits. Respiratory system examination revealed small right hemithorax and reduced intensity of breath sounds on the right side. Cardiac examination revealed prominent pulsations in right parasternal area without any murmur. His blood counts, liver

Financial or Other, Competing Interest: None.

Submission 09-02-2017, Peer Review 06-03-2017,

Acceptance 11-03-2017, Published 20-03-2017.

Corresponding Author:

Dr. V. N. Raju,

Professor

Government Hospital for Chest Diseases,

Visakhapatnam.

E-mail: durgaprasad900@gmail.com

DOI: $10.14260 /$ jemds $/ 2017 / 427$

\section{(c) (i) $\odot$}

and renal function tests were within normal limits. Chest $\mathrm{x}$ ray (fig. 1) showed small right hemithorax with shift of heart towards right side with a retrocardiac curved band like opacity extending towards diaphragm (Scimitar Sign). 2D echo showed dextroposed heart with mildly dilated RA/RV with pulmonary arterial hypertension (RVSP $=55 \mathrm{mmHg}$ ). FOB revealed a lengthy right main bronchus with missing upper lobe bronchus and the main bronchus continued to divide into a middle and lower lobe like pattern (fig. 2). On left side, branching was normal. CECT Thorax (lung window) showed a smaller right lung with no evidence of minor and major fissures, a dextroposed heart and engorged pulmonary vasculature (fig. 3). Axial section showed dilated RA and RV and scimitar vessel draining into IVC just above the diaphragm (fig. 4). Left pulmonary veins were seen draining into left atrium and similar venous drain from right side was found to be absent (fig. 5). Sagittal section showed retrocardiac vessel extending towards diaphragm (fig. 6). CT angiography with 3D reconstruction confirmed the same findings. There was no anomalous systemic arterial supply.

Based on the above imaging tests and FOB findings, the diagnosis of scimitar syndrome with unilobar right lung was made. The patient was treated with antibiotics for respiratory infection, and he was stable at the time of discharge with no further complications.

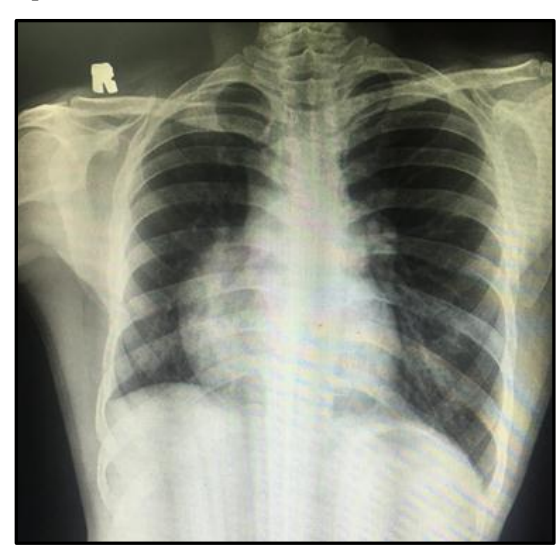

Figure 1. CXR PA View showing small right hemithorax with shift of heart towards right side with a retrocardiac curved band like opacity extending towards diaphragm (Scimitar Sign) 


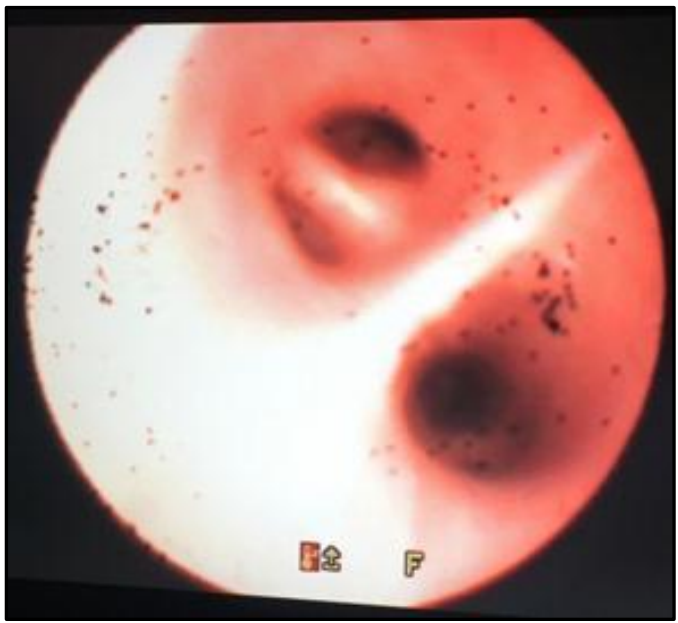

Figure 2. FOB Bronchoscopic view of right main bronchus which is seen dividing into middle lobe and lower lobe pattern

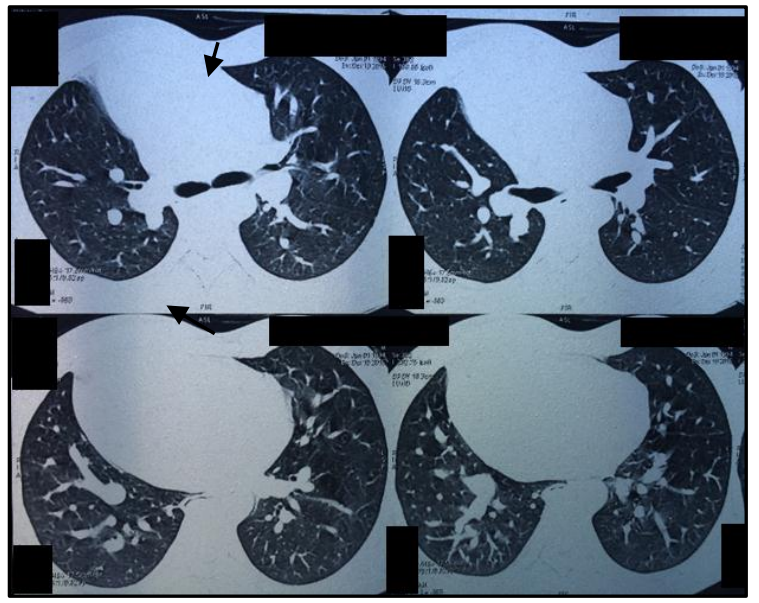

Figure 3. CT Thorax lung window showing a smaller right lung with no evidence of minor and major fissure and dextroposed heart and engorged pulmonary vasculature

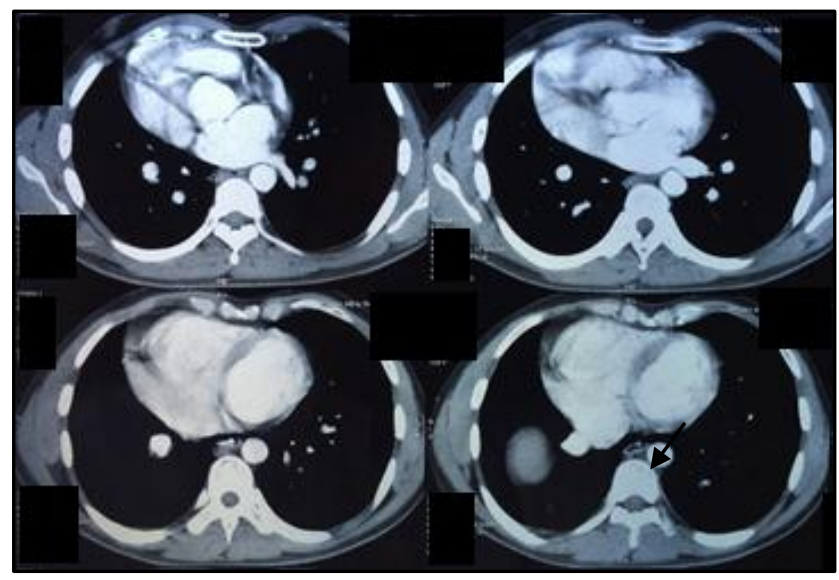

Figure 4. CECT Axial section showing dilated RA and RV and scimitar vessel draining into IVC just above the diaphragm

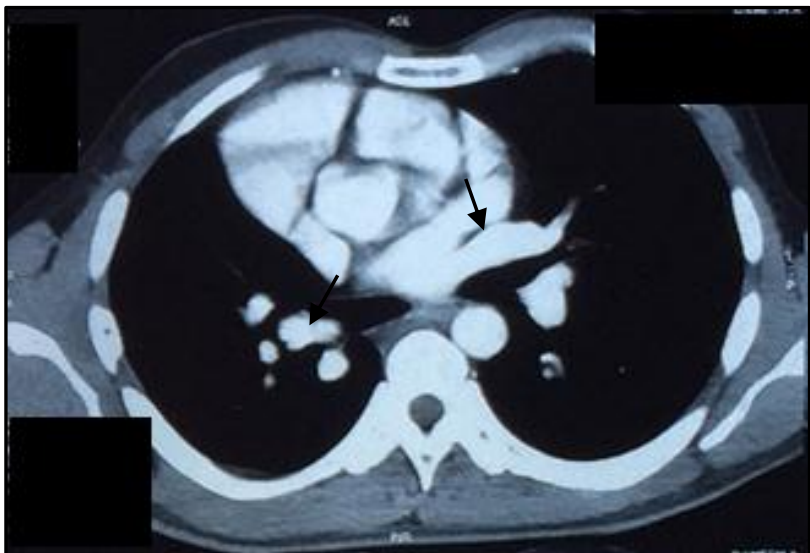

Figure 5. CECT Axial section shows left pulmonary veins draining into left atrium and similar venous drain is absent on right side

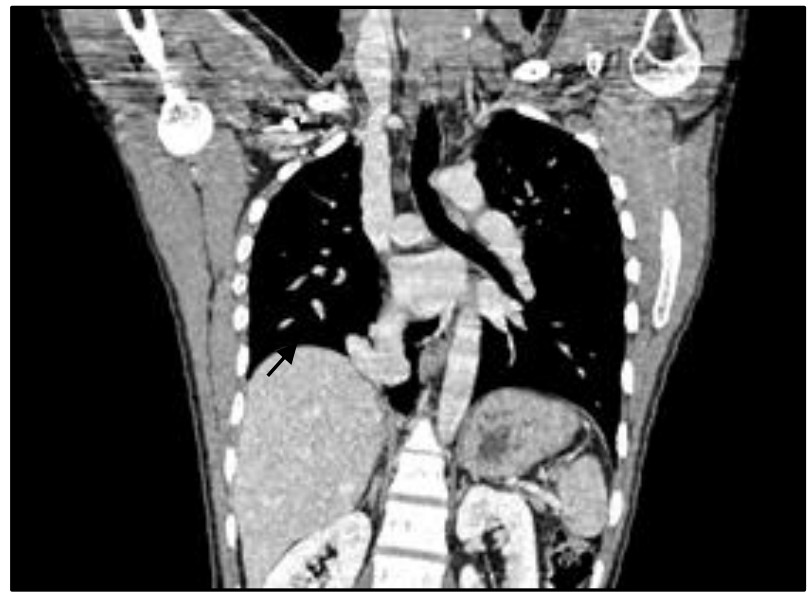

Figure 6. CECT Chest sagittal section showing retrocardiac Scimitar vessel extending towards diaphragm.

\section{DISCUSSION}

Scimitar syndrome was first described in 1836 by George Cooper during an autopsy of a 10-month-old infant. The incidence of Scimitar syndrome is $1-3 / 100,000$ live births. Scimitar Syndrome (SS) is also called as hypogenetic lung syndrome, pulmonary venolobar syndrome, epibronchial right pulmonary artery syndrome, mirror image lung syndrome, Halasz' syndrome and vena cava bronchovascular syndrome. Its main elements are-

1. Hypoplasia/lobar aplasia/agenesis involving the right lung. Abnormal lobation including bronchial isomerism with hyparterial bronchus and unilobar lung may be present. In the present case, it was a unilobar right lung. Lobar agenesia (absence of parenchyma with absent corresponding bronchus) was found in our patient. Errors of segmentation may occur and sometimes it may present as horseshoe lung.

2. An anomalous right pulmonary vein draining into the systemic venous circulation either below or above the diaphragm most commonly to the inferior vena cava, occasionally into the hepatic vein, portal vein, azygos vein, coronary sinus or the right atrium. The vein may drain the whole lung or part of it. 
3. Partial or complete anomalous systemic arterial supply from the aorta or one of its branches to the right lung with absent or hypoplastic right pulmonary artery. ASD, VSD, PDA, accessory diaphragm, and absent inferior vena cava can be other associated anomalies. Embryologically, this condition more likely represents a basic developmental derangement of entire lung bud early in embryogenesis,5,6 The reason for consistent occurrence on right side is not known.

Patients with Scimitar syndrome have variable symptoms depending on age at presentation and the degree of the left to right shunt. In infancy, the symptoms are severe due to congestive heart failure secondary to right heart volume overload. In older children and adults, it presents as recurrent respiratory infections as in the present case. On plain radiograph, small right lung with small hilum and diminished vascularity, dextroposed heart are seen. The characteristic scimitar sign consists of a broad curved, vertically oriented band like opacity along the right heart border, disappearing at the right hemidiaphragm which is the anomalous vein. It is like the shape of a Turkish sword (Scimitar). ${ }^{7}$ CECT establishes degree of lung hypoplasia and the anomalous vein with other associated anomalies and differentiates it from atelectasis, bronchopulmonary sequestration and true dextrocardia,8,9 MDCT with 3D reconstruction will detect the entire course of Scimitar vein and its eventual drainage site. 10 It also depicts anomalous arterial supply which is helpful to prevent torrential bleeding during surgery. Occasionally, a wandering right pulmonary vein may create confusion before entering normally into the left atrium. The diagnosis can also be made with MR imaging. Echocardiography should be done to rule out any associated cardiac anomalies especially atrial septal defect. Treatment of SS consists of treating respiratory tract infections with proper antibiotics and haemodynamic correction and control of bleeding in case of haemoptysis. Surgical repair consists of direct anastomosis of scimitar vein to the left atrium or creation of an ASD, transposition of anomalous vein followed by re-occlusion of ASD with a Dacron patch. Surgery is recommended when pulmonary-systemic blood flow exceeds 1.5-2 because of likelihood of progression to PAH and right ventricular failure. ${ }^{11,12}$

\section{CONCLUSION}

Scimitar syndrome is a rare congenital bronchopulmonary vascular malformation, which can be diagnosed at the earliest by radiological imaging methods like CT Angiography with 3D reconstruction or MRI. Although the Scimitar sign is visible on chest radiograph in many patients of Scimitar syndrome, additional diagnostic procedures are often necessary to confirm diagnosis and to evaluate associated abnormalities. The age of presentation and the presence of associated anomalies are important in predicting the outcome. In general, presentation in infancy and presence of heart failure are poor prognostic factors.

\section{REFERENCES}

[1] Woodring JH, Howard TA, Kanga JF. Congenital pulmonary venolobar syndrome revisited. Radiographics 1994;14(2):349-69.

[2] Kiely B, Filler J, Stone S, et al. Syndrome of anomalous venous drainage of right lung to the inferior vena cava. A review of 67 reported cases and there new cases in children. Am J Cardiol 1967;20(1):102-16.

[3] Mathey J, Galey JJ, Logeasis Y, et al. Anomalous pulmonary venous return into inferior vena cava and associated bronchovascular anomalies (the scimitars syndrome). Thorax 1968;23(4):398-407.

[4] Mordue BC. A case series of five infants with scimitars syndrome. Adv Neonatal Care 2003;3(3):121-32.

[5] Warner JO, Clements BS. Pulmonary sequestration and related congenital bronchopulmonary - vascular malformation: nomenclature and classification based on anatomical and embryological considerations. Thorax 1987;42(6):401-8.

[6] Dupuis C, Charaf LA, Breviere GM, et al. The "adult" forms of scimitars syndrome. Am J Cardiol 1992;70(4):502-7.

[7] Nazarian J, Kanne JP, Rajiah P, et al. Scimitar sign. J Thorac Imaging 2013;28(4):W61.

[8] Godwin JD, Tarver RD. Scimitar syndrome: four new cases examined with CT. Radiology 1986;159(1):1520.

[9] Olson MA, Becker GJ. The scimitar syndrome: CT findings in partial anomalous pulmonary venous return. Radiology 1986;159(1):25-6.

[10] Inove $\mathrm{T}$, Ichihara M, Uchida T, et al. Three dimensional computed tomography showing partial anomalous pulmonary venous connection complicated by the scimitar syndrome. Circulation 2002;105(5):663.

[11] Brown JW, Ruzmetov M, Minnich DJ, et al. Surgical management of scimitar syndrome: an alternative approach. J Thorac Cardiovasc Surg 2003;125(2):23845.

[12] Torres AR, Dietl CA. Surgical management of scimitar syndrome an age dependent spectrum. Cardio Vasc Surg 1993;1(4):432-8. 